\title{
Chemical Abundances of Seven Outer Halo M31 Globular Clusters from the Pan-Andromeda Archaeological Survey
}

\author{
Charli M. Sakari
}

Department of Astronomy, University of Washington, Seattle, WA 98195-1580, USA

\begin{abstract}
Observations of stellar streams in M31's outer halo suggest that M31 is actively accreting several dwarf galaxies and their globular clusters (GCs). Detailed abundances can chemically link clusters to their birth environments, establishing whether or not a GC has been accreted from a satellite dwarf galaxy. This talk presents the detailed chemical abundances of seven M31 outer halo GCs (with projected distances from M31 greater than $30 \mathrm{kpc}$ ), as derived from high-resolution integrated-light spectra taken with the Hobby Eberly Telescope. Five of these clusters were recently discovered in the Pan-Andromeda Archaeological Survey (PAndAS) - this talk presents the first determinations of integrated $\mathrm{Fe}, \mathrm{Na}, \mathrm{Mg}, \mathrm{Ca}, \mathrm{Ti}, \mathrm{Ni}, \mathrm{Ba}$, and Eu abundances for these clusters. Four of the target clusters (PA06, PA53, PA54, and PA56) are metal-poor $([\mathrm{Fe} / \mathrm{H}]<-1.5), \alpha$-enhanced (though they are possibly less alpha-enhanced than Milky Way stars at the 1 sigma level), and show signs of star-to-star $\mathrm{Na}$ and $\mathrm{Mg}$ variations. The other three GCs (H10, H23, and PA17) are more metal-rich, with metallicities ranging from $[\mathrm{Fe} / \mathrm{H}]=-1.4$ to -0.9 . While H23 is chemically similar to Milky Way field stars, Milky Way GCs, and other M31 clusters, H10 and PA17 have moderately-low [Ca/Fe], compared to Milky Way field stars and clusters. Additionally, PA17's high $[\mathrm{Mg} / \mathrm{Ca}]$ and $[\mathrm{Ba} / \mathrm{Eu}]$ ratios are distinct from Milky Way stars, and are in better agreement with the stars and clusters in the Large Magellanic Cloud (LMC). None of the clusters studied here can be conclusively linked to any of the identified streams from PAndAS; however, based on their locations, kinematics, metallicities, and detailed abundances, the most metal-rich PAndAS clusters H23 and PA17 may be associated with the progenitor of the Giant Stellar Stream, H10 may be associated with the SW Cloud, and PA53 and PA56 may be associated with the Eastern Cloud.
\end{abstract}

Keywords. galaxies: individual(M31) — galaxies: abundances — galaxies: star clusters: general - globular clusters: general — galaxies: evolution

\section{Introduction}

Globular clusters (GCs) can be used to probe the properties of their host galaxies' field stars. This is especially important for distant systems whose field stars are unresolved but whose GCs can be observed with integrated-light (IL) spectroscopy. Detailed abundances of GCs are useful for examining the formation and evolution of a galaxy, particularly whether its GCs formed in a massive galaxy or were accreted from dwarf satellites. This process of chemical tagging is possible with high-resolution IL spectroscopy, and can be applied to a variety of GC systems. This talk showcases the results from Sakari et al. (2015), who determined integrated abundances of seven outer halo $\left(R_{\text {proj }}>30 \mathrm{kpc}\right)$ M31 GCs, five of which were discovered in the Pan-Andromeda Archaeological Survey (PAndAS; McConnachie et al. 2009; Huxor et al. 2014). The location of these GCs in the outer halo, their kinematics (Veljanoski et al. 2014), and/or their projected locations along stellar streams (Mackey et al. 2010) strongly suggest an accretion scenario for many of these GCs. Detailed abundances can also be useful for determining the properties of the host dwarf galaxies. 


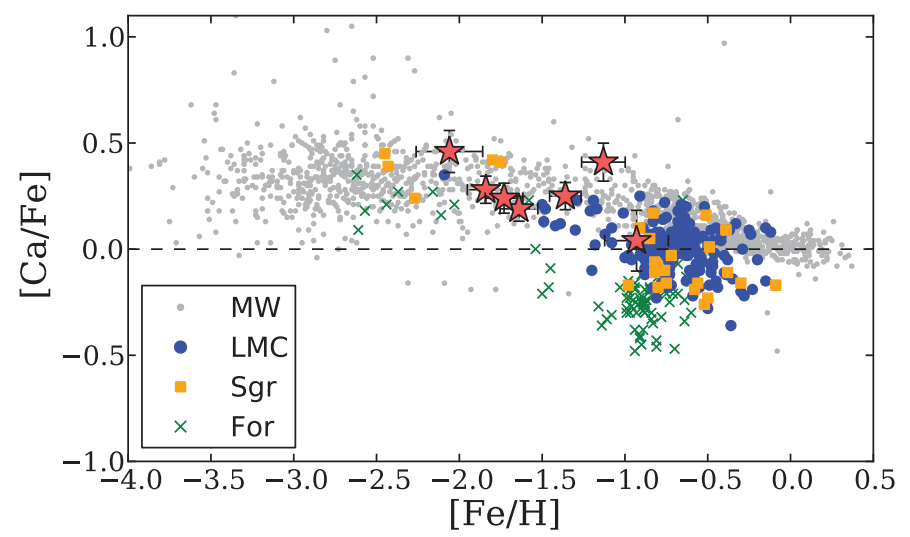

Figure 1. Comparisons of PAndAS clusters (red stars) to MW field stars (grey; from the sources in Venn et al. 2004, with supplements from Reddy et al. 2006) and dwarf galaxy field stars. LMC stars are shown with blue circles (Pompéia et al. 2008), Sgr with orange squares (Sbordone et al. 2007; Monaco et al. 2007; Carretta et al. 2010; Chou et al. 2010; McWilliam et al. 2013), and Fornax with green crosses (Tafelmeyer et al. 2010; Shetrone et al. 2003; Letarte et al. 2010). The error bars show the random errors. The dashed horizontal line shows the solar value.

\section{Integrated Chemical Abundances of Outer Halo M31 GCs}

Spectra of the seven target GCs were obtained with the High Resolution Spectrograph on the Hobby-Eberly Telescope, yielding a spectral resolution of $R=30000$ (see Sakari et al. 2015 for details). S/N ratios ranged from $\sim 40-140$ per resolution element. Hubble Space Telescope partially resolved photometry (down to the horizontal branch) was used to constrain the ages and metallicities of the GC stellar populations; the final adopted ages and metallicities were then determined spectroscopically (see McWilliam \& Bernstein 2008; Colucci et al. 2014; Sakari et al. 2013). BaSTI isochrones (Pietrinferni et al. 2004) were adopted to model the underlying populations. Integrated abundances were determined with the ILABUNDS code (McWilliam \& Bernstein 2008).

Detailed abundances of a variety of elements ( $\mathrm{Fe}, \mathrm{Na}, \mathrm{Mg}, \mathrm{Ca}, \mathrm{Ti}, \mathrm{Ni}, \mathrm{Ba}$, and $\mathrm{Eu}$ ) are given in Sakari et al. (2015). Figure 1 shows integrated $[\mathrm{Ca} / \mathrm{Fe}]$ versus $[\mathrm{Fe} / \mathrm{H}]$ in the PAndAS GCs compared to the abundances from individual field stars in the Milky Way and various satellite galaxies. Note that the GC Ca abundances follow the chemical evolution profile of field stars that formed in the same birth environment (e.g., Pritzl et al. 2005; Sakari et al. 2013; Colucci et al. 2014; Hendricks et al. 2016); the integrated $[\mathrm{Ca} / \mathrm{Fe}]$ is therefore a useful abundance ratio for chemical tagging purposes. $\dagger$ Several of the PAndAS GCs have low $[\mathrm{Ca} / \mathrm{Fe}]$ (and other ratios, such as high $[\mathrm{Ba} / \mathrm{Eu}]$ ) indicating that they likely formed in dwarf galaxies that are currently being accreted by M31. The abundances themselves are also useful for constraining the mass of the progenitor dwarf galaxies - for example, PA17 (the most metal-rich target) has abundances that are more consistent with a galaxy like the Large Magellanic Cloud (LMC) or the Sagittarius dwarf spheroidal (Sgr) than with the Fornax dwarf (For).

$\dagger$ Other integrated GC abundances may not follow the field stars, e.g., Na and Mg. These abundances are useful for probing the nature of GCs (see Sakari et al. 2013, 2015; Colucci et al. 2014). 


\section{The Nature of M31's Outer Halo}

These detailed abundances can be combined with kinematic and spatial information to link GCs with specific progenitor streams and/or other GCs. PA17's high $[\mathrm{Fe} / \mathrm{H}]$ and low $[\mathrm{Ca} / \mathrm{Fe}]$ suggest that it may be associated with the metal-rich Giant Stellar Stream (GSS; see, e.g., Ibata et al. 2014) - though it does not lie along the GSS, models suggest that the stream may have multiple wraps around M31 (Fardal et al. 2013; Kirihara et al. 2016). The moderate-metallicity GC H10 lies on an extension of the SW Cloud, and has similar abundances and kinematics to its GCs (Mackey et al. 2013). H10's [Ca/Fe] implies that the SW Cloud progenitor likely had a similar mass as Sgr. Finally, the more metal-poor GCs PA53 and PA56 have similar abundances to each other, plus similar kinematics to two GCs that lie along the nearby Eastern Cloud (McMonigal et al. 2016). The abundances of PA53 and PA56 indicate that the progenitor of the Eastern Cloud was likely similar to the Fornax dwarf galaxy.

Together, the combination of resolved photometry and IL spectroscopy has demonstrated that M31's outer halo is at least partially been built up by the accretion of satellite dwarfs. Studies of the outer halo field stars and the GCs have provided essential information about the masses of the satellites that are being accreted to form M31's outer halo. This level of detail will be difficult to achieve in more distant galaxies, particularly when the field stars cannot be resolved. When resolved photometry is no longer feasible, IL spectroscopy of GCs remains a powerful tool for unraveling the assembly histories for distant, unresolved systems.

\section{References}

Carretta, E., Bragaglia, A., Gratton, R., et al. 2010, A\&A, 520, 95

Chou, M.-Y., Cunha, K., Majewski, S. R., et al. 2010, ApJ, 708, 1290

Colucci, J. E., Bernstein, R. B., \& Cohen, J. G. 2014, ApJ, 797, 116

Fardal, M. A., Weinberg, M. D., Babul, A., et al. 2013, MNRAS, 434, 2779

Hendricks, B., Boeche, C., Johnson, C. I., et al. 2016, A 6 A, 585, 86

Huxor, A. P., Mackey, A. D., Ferguson, A. M. N., et al. 2014, MNRAS, 442, 2165

Ibata, R. A., Lewis, G. F., McConnachie, A. W., et al. 2014, ApJ, 780, 128

Kirihara, T., Miki, Y., Mori, M., \& Kawaguchi, T. 2016, arXiv:1603.02682

Letarte, B., Hill, V., Tolstoy, E., et al. 2010, A\&A A, 523, 17

Mackey, A. D., Huxor, A. P., Ferguson, A. M. N., et al. 2010, ApJ, 717, 11

Mackey, A. D., Huxor, A. P., Ferguson, A. M. N., et al. 2013, MNRAS, 429, 281

McConnachie, A. W., et al. 2009, Nature, 461, 66

McMonigal, B., Bate, N. F., Conn, A. R., et al. 2016, MNRAS, 456, 405

McWilliam, A. \& Bernstein, R. 2008, ApJ, 684, 326

McWilliam, A., Wallerstein, G., \& Mottini, M. 2013, ApJ, 778, 149

Monaco, L., Bellazzini, M., Bonifacio, P., et al. 2007, A\&SA, 464, 201

Pietrinferni, A., Cassisi, S., Salaris, M., \& Castelli, F. 2004, ApJ, 612, 168

Pompéia, L., Hill, V., Spite, M., et al. 2008, A\&A, 480, 379

Pritzl, B. J., Venn, K. A., \& Irwin, M. 2005, AJ, 130, 2140

Reddy, B. E., Lambert, D. L., \& Prieto, C. A. 2006, MNRAS, 367, 1329

Sakari, C. M., Shetrone, M., Venn, K., McWilliam, A., \& Dotter, A. 2013, MNRAS, 434, 358

Sakari, C. M., Venn, K., Shetrone, M., Dotter, A., \& Mackey, D. 2014, MNRAS, 443, 2285

Sakari, C. M., Venn, K. A., Mackey, D., et al. 2015, MNRAS, 448, 1314

Sbordone, L., Bonifacio, P., Buonanno, R., et al. 2007, A\&A, 465, 815

Shetrone, M., Venn, K., Tolstory, E., et al. 2003, AJ, 125, 684

Tafelmeyer, M., Jablonka, P., Hill, V., et al. 2010, A\&A, 524, A58

Veljanoski, J., Mackey, A. D., Ferguson, A. M. N., et al. 2014, MNRAS, 442, 2929

Venn, K. A., Irwin, M., Shetrone, M. D., et al. 2004, AJ, 128, 1177 